\title{
Impact of Extraischemic Hemorrhage After Thrombolysis with Intravenous Recombinant Tissue Plasminogen Activator
}

\author{
Takuro Endo ${ }^{1}$, Taizen Nakase ${ }^{2}$, Masahiro Sasaki ${ }^{2}$ and Tatsuya Ishikawa ${ }^{1}$ \\ ${ }^{1}$ Department of Neurological Surgery, Research Institute for Brain and Blood Vessels, Akita, Japan \\ ${ }^{2}$ Department of Stroke Science, Research Institute for Brain and Blood Vessels, Akita, Japan
}

Corresponding author: Dr. Taizen Nakase, Department of Stroke Science, Research Institute for Brain and Blood Vessels, Akita, 6-10 Sensyu Kubota Machi, Akita, 010-0874, Japan, Tel: +81-18-833-0115; Fax: +81-18-833-2104; E-mail: nakase@akita-noken.jp

Received: Jun 15, 2016; Accepted: Jul 21, 2016; Published: Jul 25, 2016

Citation: Endo T, Nakase T, Sasaki M, et al. Impact of Extraischemic Hemorrhage After Thrombolysis with Intravenous Recombinant Tissue Plasminogen Activator. J Neurol Neurosci. 2016, 7: S3.

\section{Abstract}

Background: Thrombolysis is efficacious for acute ischemic stroke; however it might increase the risk of following hemorrhage. Generally, hemorrhage may occur within the ischemic region because of ischemiareperfusion injury. However, hemorrhage of extraischemic lesion called extraischemic hemorrhage (EH) may sometimes observed. The risks and outcome of $\mathrm{EH}$ have been still controversial. Therefore, this study aimed to reveal the clinical characteristics of $\mathrm{EH}$ in recent cases.

Methods: Acute ischemic stroke patients who were treated with intravenous recombinant tissue plasminogen activator administration within $4.5 \mathrm{hrs}$ following onset were consecutively screened $(n=112)$. All cases were classified into no-bleeding (NB), cerebral hemorrhage within infarct lesion ( $\mathrm{HI}$ ) and $\mathrm{EH}$ groups. The MRI findings, patient's background and outcome were assessed between $\mathrm{EH}$ and other groups.

Results: Incidence rate of $\mathrm{NB}, \mathrm{HI}$ and $\mathrm{EH}$ were $62.5 \%$, $31.2 \%$ and $6.3 \%$, respectively. There was no significant difference of patients' background between $\mathrm{EH}$ and other groups. While, patients of EH group showed significantly higher frequency of microbleeds in subcortical region compared with NB group ( $p=0.01: 71.4 \%$ and $21.4 \%$, respectively). Leukoaraiosis in deep white matter region was significantly severer in EH compared with NB group $(p=0.03)$. Although neurologic severity at onset was the same among three groups, the outcome was significantly worse in $\mathrm{EH}$ group compared with other groups $(\mathrm{p}=0.04)$.

Conclusion: Our results confirmed that extraischemic hemorrhage after thrombolysis could cause worse outcome. Moreover, it was revealed the underlying small vessel disease, represented by microbleeds and leukoaraiosis in subcortical area, might be associated with extraischemic hemorrhage.

Keywords: Ischemic stroke; Treatment; Thrombolysis; Hemorrhagic change; Outcome; Complication

\section{Introduction}

Thrombolytic therapy with intravenous recombinant tissue plasminogen activator (IV rt-PA) administration is efficacious treatment for acute ischemic stroke within 4.5 hours of the onset, and also is recommended as best therapy in clinical guideline for stroke in Japan [1]. However, hemorrhagic complication is sometimes observed following the thrombolysis, and it might affect disease state and prognosis. Trouillas reported that cerebral hemorrhage after thrombolysis could be classified into three types, i.e., hemorrhagic infarction $(\mathrm{HI})$, parenchymal hemorrhage and extraischemic hematoma (EH) [2]. Particularly, the incidence rate of $\mathrm{EH}$ is very low, and it's pathogenesis and prognosis has not been fully understood. In this study, we evaluated the possible association between the incidences of $\mathrm{EH}$ and the pathology of cerebral small vessels by acquired imaging findings. Furthermore, we explored the premorbid clinical settings and outcome of the patients who suffered from $\mathrm{EH}$ after thrombolysis.

\section{Patients and Methods}

Following the approval of the ethical committee of the Research Institute for Brain and Blood Vessels -Akita, consecutive acute ischemic stroke patients who were admitted to our hospital and treated with IV rt-PA within 4.5 hours of onset were retrospectively screened between April 1, 2007 and March 31, 2015. We excluded patients who were not performed magnetic resonance imaging (MRI) scan because of implantation of a pacemaker, joint replacement and old intracranial clips. Moreover, we excluded patients who underwent intra-arterial thrombolysis and mechanical thrombectomy following IV rt-PA administration (Figure 1). The Japanese guideline was adopted for the indication of IV rt-PA administration [1]. Blood pressure was controlled by IV $\mathrm{Ca}$ channel blocker and maintained systolic blood pressure (sBP) $<185 \mathrm{mmHg}$ and diastolic blood pressure $<110 \mathrm{mmHg}$ before 
IV rt-PA infusion, and sBP $<180 \mathrm{mmHg}$ and $\mathrm{dBP}<105 \mathrm{mmHg}$ following 24 hours of infusion. Within 24 hours of thrombolysis, any other antithrombotic agents were not prescribed.

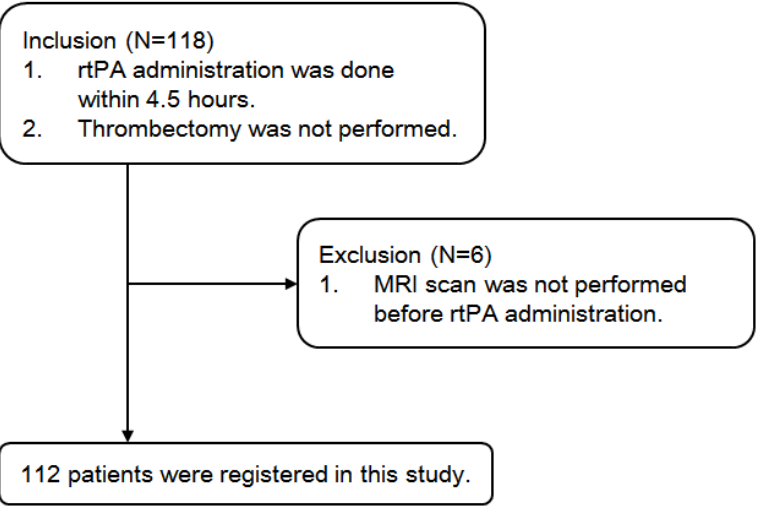

Figure 1 Flowchart of patients' selection.

All patients were taken MRI examination at 24 hours after thrombolysis. Then, they were classified into three groups depending on MRI T2* weighted image (TR 3000, TE 50); nonbleeding group (NB group), hemorrhagic infarction group (HI group) and extraischemic hemorrhage group (EH group). In this study, both parenchymal and petechial hemorrhages were defined as $\mathrm{HI}$ unless the hemorrhage was observed within the ischemic lesion. We compared between $\mathrm{EH}$ group and other two groups for identifying patient characteristics in $\mathrm{EH}$ group.

\section{Patient background}

Stroke risks such as hypertension, diabetes mellitus, hyperlipidemia, smoking, drinking and past ischemic stroke were assessed based on the clinical record. National Institute of Health stroke scale (NIHSS) was used for evaluation of neurologic severity. Modified Rankin scale (mRS) at the time of discharge from the hospital was used for evaluation of outcome. Good clinical outcome was defined as $m R S<3$, because it is one of the important point whether activity of daily life was independent or not. In addition, mRS was compared between at the time of admission and discharge for evaluating how patient's outcome was affected by hemorrhagic complications.

\section{Cerebral microbleeds (CMBs)}

CMBs were assessed based on MRI $\mathrm{T} 2 * \mathrm{WI}$, which was undertaken at the time of admission. Lesions within cerebral parenchyma were classified into three regions; infratentrial, basal ganglia and subcortical regions.

\section{Leukoaraiosis}

Leukoaraiosis was assessed based on MRI T2 WI (TR 4000, TE 100) and fluid attenuated inversion recovery (FLAIR) image
(TR 10000, TE 100, TI 2200), which were undertaken at the time of admission. Following the guideline of the Japan brain dock society, severity of leukoaraiosis was classified into 5 categories (0: absent, 1: small, 2: mild, 3: moderate and 4: severe) in two regions; periventricular hyperintensity (PVH) and deep and subcortical white matter hyperintensity (DSWMH) [3].

\section{Statistical analysis}

All statistical analysis was performed by JMP version 9 software (SAS institute Inc., NC). Numerical data was presented as median and quartile. Categorical data was presented as percentage. NIHSS at the time of admission and the severity of leukoaraiosis between EH and other two groups were evaluated by Steel-Dwass test. Other factors were evaluated by Fisher's exact test. Improvement of mRS from admission to discharge was assessed as patient's prognosis.

\section{Results}

\section{Patients' background}

Patients of 70 (62.5\%) showed no hemorrhagic transformation after thrombolysis (NB group). The incidence rate of hemorrhagic change after thrombolysis was 35 (31.2\%) and $7(6.3 \%)$ in $\mathrm{HI}$ and $\mathrm{EH}$ groups, respectively. Furthermore, hemorrhage with mass effect was 31 and 4 cases, and without mass effect was 4 and 3 cases in $\mathrm{HI}$ and $\mathrm{EH}$ groups, respectively. Regarding the stroke risks, there was no significant difference between EH and NB or HI groups (Table 1). Neurologic severity was not different among three groups. The stroke subtypes of all patients were cardiogenic embolism $(n=77)$, atherothrombotic infarction $(n=21)$, lacunar infarction $(n=9)$ and unknown origin $(n=5)$. There was no lacunar infarction and unknown origin infarction in $\mathrm{EH}$ group. The distribution of stroke subtypes was not significantly different among three groups (Figure 2).

\section{$\mathrm{CMBs}$ and leukoaraiosis}

As shown in Table 2, percentage of the existence of CMBs in the subcortical region was significantly higher in $\mathrm{EH}$ compared with NB groups ( $p=0.01 ; 71.4 \%$ and $21.4 \%$, respectively). EH group showed significantly severer DSWMH compared with NB group $(p=0.03)$. The severity of PVH was not different among three groups.

\section{Outcome}

There was no difference of mean mRS score at discharge among three groups (Table 3). However, the frequency of patients in poor outcome (mRS 3 - 5) was significantly higher in EH group compared with NB and HI groups ( $p=0.04: 100 \%$, $57.1 \%$ and $57.1 \%$, respectively). Moreover, number of patients who showed the improvement of mRS score was significantly 
fewer in $\mathrm{EH}$ group (14.3\%) compared with NB (60.0\%) and $\mathrm{HI}$ groups (74.3\%) ( $P=0.04$ and $P<0.01$, respectively).

Table 1 Patient background in each group.

\begin{tabular}{|c|c|c|c|c|c|}
\hline & EH group & NB group & $P$ value (EH vs NB) & HI group & $P$ value (EH vs $\mathrm{HI})$ \\
\hline $\mathrm{n}$ & 7 & 70 & & 35 & \\
\hline Age, median (range) & $78(68-80.5)$ & $74(63-80)$ & 0.75 & $72(56.5-79)$ & 0.63 \\
\hline Sex (male/female) & 3-Apr & $46 / 24$ & 0.69 & $27 / 8$ & 0.35 \\
\hline Hospitalization, median (range) & $36(19.5-42)$ & $28(15-40)$ & 0.8 & $22(17.5-34)$ & 0.72 \\
\hline Hypertension & $4(57.1 \%)$ & $31(44.2 \%)$ & 0.7 & $16(45.7 \%)$ & 0.69 \\
\hline Hyperlipidemia & $3(42.9 \%)$ & $22(31.4 \%)$ & 0.68 & $3(8.6 \%)$ & 0.16 \\
\hline Diabetes mellitus & $2(28.6 \%)$ & $17(24.3 \%)$ & 1 & $4(11.4 \%)$ & 0.26 \\
\hline History of stroke & $0(0 \%)$ & $12(17.1 \%)$ & 0.59 & $6(17.1 \%)$ & 0.57 \\
\hline Antithrombotic therapy & $4(57.1 \%)$ & $18(25.7 \%)$ & 0.1 & $10(28.6 \%)$ & 0.2 \\
\hline Smoking & $1(14.3 \%)$ & $21(30.0 \%)$ & 0.67 & $15(42.9 \%)$ & 0.22 \\
\hline Drinking & $2(28.6 \%)$ & $29(41.4 \%)$ & 0.7 & $17(48.6 \%)$ & 0.43 \\
\hline NIHSS at admission, & $8(7-17.5)$ & $12(9-17)$ & 0.68 & $14(9-18.5)$ & 0.69 \\
\hline
\end{tabular}

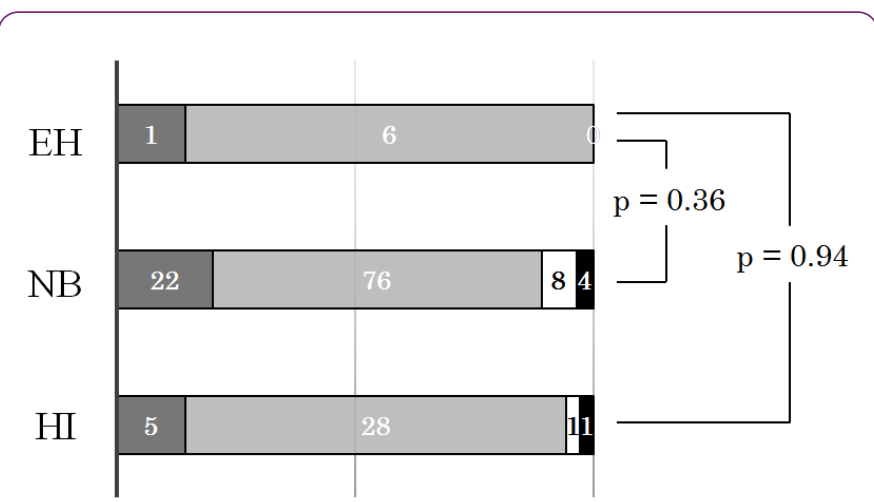

Figure $\mathbf{2}$ The distribution of stroke subtypes in each group. There was no significant difference among three groups. Dark gray, light gray, white and black bars indicate atherothrombotic infarction, cardiogenic embolism, lacunar infarction and unknown origin. Numbers in each bar stand for the number of patients in each group.

\section{Discussion}

Our results confirmed that the extraischemic hemorrhage might adversely affect a patient's prognosis. Then, we revealed that the presence of CMBs in subcortical region and severity of DSWMH could be useful predictors of extraischemic hemorrhage after IV rt-PA administration.

Hemorrhagic complications after thrombolysis have been reported to be classified into three types, hemorrhagic infarction, parenchymal hematoma and extraischemic hemorrhage, according to imaging findings and pathophysiology [2]. It is reported that incidence of cerebral hemorrhage in extraischemic region is $1.3-3.7 \%$ [2,4-6], and it is still undetermined the risk factors which relate to extraischemic hemorrhage [5-8]. In our study, there was not any significant difference of backgrounds which might relate to the extraischemic hemorrhage, suggesting that clinical risks would not be helpful to predict the extraischemic hemorrhage. Meanwhile, the hemorrhagic transformation of ischemic lesion has been reported to associate to the existence of arterial fibrillation, suggesting that cardiogenic embolism could be a cause of hemorrhagic infarction [6]. However, there has been no report which suggests the association between stroke subtypes and incidence of extraischemic hematoma.

It has been pointed out that incidence of extraischemic hemorrhage is associated to vascular fragility, which is pathologically explained by small vessel disease, observed as CMBs or leukoaraiosis in MRI findings. CMBs might associate with hypertensive microangiopathy in basal ganglia region and cerebral amyloid angiopathy (CAA) in subcortical region [9-12]. Then, presence of $\mathrm{CMBs}$ is reported to associate with incidence of cerebral hemorrhage in both healthy individuals and patients treated with antithrombotic agents [12-15]. Multiple CMBs, especially in CAA, could also relate to the increased risk of cerebral hemorrhage $[16,17]$. On the other hand, some studies reported that presence of $\mathrm{CMBs}$ did not relate to cerebral hemorrhage following thrombolysis [18-20]. In this study, CMBs in subcortical region was significantly frequent in $\mathrm{EH}$ group than NB group. It can be said that the 
presence of $\mathrm{CMBs}$ could be associated with $\mathrm{EH}$ after thrombolysis.

Table 2 The number of patients with CMBs and the severity of leukoaraiosis in each region.

\begin{tabular}{|c|c|c|c|c|c|}
\hline & EH group & NB group & $P$ value (EH vs NB) & HI group & $P$ value (EH vs $\mathrm{HI})$ \\
\hline \multicolumn{6}{|l|}{ CMBs } \\
\hline Infratentrial & $3(42.9 \%)$ & $12(17.1 \%)$ & 0.13 & $12(34.3 \%)$ & 0.69 \\
\hline Basal ganglia & $5(71.4 \%)$ & $21(30.0 \%)$ & 0.04 & $13(37.1 \%)$ & 0.12 \\
\hline Subcortical & $5(71.4 \%)$ & $15(21.4 \%)$ & 0.01 & $12(34.3 \%)$ & 0.1 \\
\hline \multicolumn{6}{|l|}{ Leukoraiosis } \\
\hline DSWMH Median (range) & $3(2-4)$ & $2(1-2)$ & 0.03 & $2(1-2)$ & 0.17 \\
\hline PVH Median (range) & $2(1-3.5)$ & $1(0-2)$ & 0.11 & $1(0-2)$ & 0.07 \\
\hline
\end{tabular}

Table 3 Comparison of $\mathrm{mRS}$ at the time of admission and discharge in each group.

\begin{tabular}{|c|c|c|c|c|c|}
\hline & EH group & NB group & $P$ value (EH vs NB) & HI group & $P$ value (EH vs $\mathrm{HI})$ \\
\hline $\mathrm{mRS}$ at discharge median (range) & $4(3.5-5)$ & $4(1-4)$ & 0.34 & $3(2-4)$ & 0.51 \\
\hline \multicolumn{6}{|l|}{ Number of patients } \\
\hline $0-2$ & 0 & 30 & & 15 & \\
\hline $3-6$ & 7 & 40 & 0.04 & 20 & 0.04 \\
\hline \multicolumn{6}{|c|}{ Comparison of $\mathrm{mRS}$ from admission to discharge } \\
\hline Improved & 1 & 42 & 0.04 & 26 & $<0.01$ \\
\hline Unchanged/Worsened & 6 & 28 & & 9 & \\
\hline
\end{tabular}

Leukoaraiosis also reflects small vessel disease, which is formed by microvascular degeneration and deep white matter ischemia caused by such as hypertension and diabetes mellitus $[9,21]$. Moreover, leukoaraiosis can be assumed to suggest a disorder of blood brain barrier (BBB) in the cerebrum. However, according to previous reports, it is still controversial about the association between severity of leukoaraiosis and cerebral hemorrhage after thrombolysis [22-26]. Herein, this study showed the significant correlation between extraischemic hemorrhage after thrombolysis and severity of leukoaraiosis, especially about DSWMH, supporting the previous reports in which severity of not PVH but DSWMH had significant correlation with cerebral hemorrhage after thrombolysis [23,24].

It has been reported that developing extraischemic hemorrhage adversely affect a patient's prognosis $[4,5,15,16]$. While, pathogenesis of poor outcome of extraischemic hemorrhage has not been unveiled. In this study, the incidence of $\mathrm{HI}$ was $31 \%$ and most of them showed parenchymal hemorrhage. The incidence of $\mathrm{EH}$ was $6.3 \%$ and the rate of hemorrhage with mass effect and without mass effect was the same. Nevertheless, patients with extraischemic hemorrhage showed not only worse outcome but also poor improvement during hospitalization. Possibly, hemorrhagic damage might induce neurologic deterioration addition to the original deficit caused by ischemia.
There are some limitations. This study was retrospective and single-center study. The incidence of extraischemic hemorrhage was only observed within $24 \mathrm{hr}$ following thrombolytic therapy. To ensure our results, a larger prospective study should be conducted. Nevertheless, it is worthwhile to report and accumulate data of the clinical characteristics of extraischemic hemorrhage, since the incidence of extraischemic hemorrhage is very low.

\section{Conclusion}

According to imaging findings on $\mathrm{MRI}, \mathrm{CMBs}$ and leukoaraiosis could be risk factors of extraischemic hemorrhage following rt-PA thrombolysis. Extraischemic hemorrhage might be associated with vascular fragility in small arteries of cerebrum. Moreover, because extraischemic hemorrhage related to the poor outcome, it should be aware of during acute phase treatment.

\section{Contribution of Each Author}

TE conducted throughout this study. TN carried out the statistical analysis and manuscript preparation. MS performed the screening of patients. TI coordinated and advised the settings of this study. 


\section{Conflict of Interest}

All authors report no financial or institutional conflicts in this study.

\section{Reference}

1. Minematsu K, Toyoda K, Hirano T (2013) Guidelines for the intravenous application of recombinant tissue-type plasminogen activator (alteplase), the second edition, October 2012: a guideline from the Japan Stroke Society. J Stroke Cerebrovasc Dis 5: 571-600.

2. Trouillas P, Von Kummer R (2006) Classification and pathogenesis of cerebral hemorrhage after thrombolysis in ischemic stroke. Stroke 37: 556-561.

3. Kobayashi S (2014) Japan brain dock society: Guidelines for the brain dock 2014. Sapporo: Kyobunsha Inc.

4. The NINDS t-PA stroke study group (1997) Intracerebral hemorrhage after intravenous t-PA therapy for ischemic stroke. Stroke 28: 2109-2118.

5. Maritinez HE, Martinez PS, Delgado MR (2010) Remote cerebral hematomas in patients treated with intravenous rt-PA. J Neurol 257: 1062-1066.

6. Mazya MV, Ahmed N, Ford GA (2014) Remote or extraischemic intracerebral hemorrhage - An uncommon complication of stroke thrombolysis. Stroke 45: 1657-1663.

7. Ruecker M, Matosevic B, Willeit P (2012) Sub-therapeutic warfarin therapy entails an increase bleeding risk after stroke thrombolysis. Neurology 79: 31-38.

8. Furuya D, Tanahashi N (2007) Clinical characteristics of intracranial hemorrhage after intravenous thrombolysis with rt-PA. Jpn J Stroke 29: 799-804.

9. Onodera $O$ (2011) What is cerebral small vessel disease? Clin Neurol 51: 399-405.

10. Tanaka A, Ueno Y, Nakayama $Y$ (1999) Small chronic hemorrhages and ischemic lesions in association with spontaneous intracerebral hematomas. Stroke 30: 1637-1642.

11. Imaizumi T (2013) The origin and clinical importance of cerebral microbleeds. Jpn J Stroke 35: 397-405.

12. Bokura H, Saika R, Yamaguchi T (2011) Microbleeds are associated with subsequent hemorrhage and ischemic stroke in healthy elderly individuals. Stroke 42: 1867-1871.

13. Soo YOY, Yang SR, Lam WWM (2008) Risk vs. benefit of anti-thrombotic therapy in ischemic stroke patients with cerebral microbleeds. J Neurol 255: 1679-1686.
14. Kouge J, Hirooka S, Noda K (2012) A case of intracerebral hemorrhage at the site of cerebral microbleeds after IV rtPA thrombolysis for cerebral infarction. Jpn J Stroke 34: 94-97.

15. Yamauchi T, Katsumura H, Noguchi $Y$ (2013) Cerebral hematoma at the site of cerebral infarction and extraischemic cerebral hematoma in the temporal lobe after intravenous rt-PA thrombolysis for cerebral infarction: a case report. Jpn J Stroke 35: 448-452.

16. Takezawa H, Imai K, Hamanaka M (2010) Extraischemic cerebral hematomas of the cerebellum in extremely elderly stroke patient treated with intravenous rt-PA therapy: A case report. Jpn J Stroke 32: 379-383.

17. McCarron M, Nicoll JAR (2004): Cerebral amyloid angiopathy and thrombolysis related intracerebral hemorrhage. Lancet Neurol 3: 484-492.

18. Kim HS, Lee DH, Ryu CW (2006) Multiple cerebral microbleeds in hyperacute ischemic stroke: impact on prevalence and severity of early hemorrhagic transformation after thrombolytic treatment. Am J Radiol 186: 1443-1449.

19. Drex L, Nighoghossian N, Hermier M (2004) Thrombolysis for ischemic stroke in patients with old microbleeds on pretreatment MRI. Cerebrovasc Dis 17: 238-241.

20. Kakuda W, Thijs VN, Lansberg MG (2005) Clinical importance of microbleeds in patients receiving IV thrombolysis. Neurology 65: 1175-1178.

21. Pantoni L, Garcia JH (1997) Pathogenesis of leukoaraiosis. Stroke 28: 652-659.

22. Singer OC, Humpich MC, Fiehler J (2008) Risk for symptomatic intracerebral hemorrhage after thrombolysis assessed by diffusion-weighted magnetic resonance imaging. Ann Neurol 63: 52-60.

23. Neumann HT, Hoelig S, Berkefeld J (2006) Leukoaraiosis is a risk factor for symptomatic intracerebral hemorrhage after thrombolysis for acute stroke. Stroke 37: 2463-2466.

24. Shi ZS, Loh Y, Liebeskind DS (2012) Leukoaraiosis predicts parenchymal hematoma after mechanical thrombectomy in acute ischemic stroke. Stroke 43: 1806-1811.

25. Demchuk AM, Khan F, Hill MD (2008) Importance of leukoaraiosis on CT for tissue plasminogen activator decision making: evaluation of the NINDS rt-PA stroke study. Cerebrovasc Dis 26: 120-125.

26. Aries MJ, Uyttenbooogaart M, Vroomen PC (2010) tPA treatment for acute ischemic stroke in patients with leukoaraiosis. Eur J Neurol 17: 866-870. 